J. Nonlinear Var. Anal. 4 (2020), No. 2, pp. 225-239

Available online at http://jnva.biemdas.com

https://doi.org/10.23952/jnva.4.2020.2.05

\title{
CONVEX DECOMPOSITIONS AND THE VALENCE OF SOME FUNCTIONS
}

\author{
CORNEL PINTEA $^{1, *}$, ADRIAN TOFAN $^{2}$ \\ Department of Mathematics, Faculty of Mathematics and Computer Science, \\ Babeş-Bolyai University, Str. Kogălniceanu no. 1, 400084 Cluj-Napoca, Romania
}

\begin{abstract}
The aim of this paper is twofold. On one hand, we provide examples of $\mathbb{R}^{n}$-valued functions on some open subsets $D$ of $\mathbb{R}^{n}$ whose restrictions to the convex subsets of $D$ are all injective. Such applications are shortly called CIP functions. On the other hand, we provide alternative descriptions of the maximal convex subsets of the convex open sets with compact convex subsets removed. The maximal convex subsets of $\mathbb{R}^{n}$ with convex sets removed were characterized before by Martínez-Legaz and Singer [Compatible Preorders and Linear Operators on $\mathbb{R}^{n}$, Linear Algebra Appl. 153 (1991), 53-66] as being the convex subsets of $\mathbb{R}^{n}$, shortly called hemispaces, whose complements are convex too. The two topics merge together as the smallest number $k$ of convex subsets, of the considered open set, needed to cover it, is an upper bound for the valence of every CIP function on that open set.
\end{abstract}

Keywords. Convex decompositions; CIP functions; Maximal convex sets

\section{INTRODUCTION}

The importance of convex decompositions of a certain open set $D \subseteq \mathbb{R}^{n}$ is emphasized here through the valence of every function $F: D \longrightarrow \mathbb{R}^{n}$ whose restrictions to the convex subsets of $D$ are all injective. Such applications are said to satisfy the convex injectivity property (CIP) and we call them CIP-functions. Indeed, every convex decomposition of $D$ provides, through the number of its convex components, an upper bound for the cardinalities of all inverse images of such a function $F: D \longrightarrow \mathbb{R}^{n}$. More precisely the number of solutions of any equation $F(x)=y$, where $F: D \longrightarrow \mathbb{R}^{n}$ is a CIP-function and $y \in \mathbb{R}^{n}$, does not exceed the number of convex components of any covering of $D$ by convex subsets. They arise if we remove the convexity assumption in a global injectivity criteria for functions defined on convex sets. Note that $F: D \longrightarrow \mathbb{R}^{n}$ is a CIP-function if and only if its restriction to every convex open subset of $D$ is injective, as every segment $[x y] \subseteq D$ is actually contained in a convex open subset of $D$. It is therefore worth to consider the minimum cardinal number $k:=\gamma(D)$, usually called the convexity number of $D$, such that $D$ can be covered by $k$ convex subsets of $D$, as the valence of every CIP function $F: D \longrightarrow \mathbb{R}^{n}$ is at most $\gamma(D)$.

Note that $\gamma(D) \leq \boldsymbol{\aleph}_{0}$, as $\mathbb{R}^{n}$ is a second-countable space and each of its open subset is, for instance, a countable union of open balls. The openness of $D$ is essential for $\gamma(D)$ to be at

${ }^{*}$ Corresponding author.

E-mail addresses: cpintea@math.ubbcluj.ro (C. Pintea), adriantofanioan@yahoo.com (A. Tofan).

Received January 24, 2020; Accepted July 7, 2020.

(C)2020 Journal of Nonlinear and Variational Analysis 
most countable as many closed subsets of $\mathbb{R}^{n}$, such as the unit sphere $S^{n-1}$ when $n \geq 2$, are not countably convex (see also [7]).

Several convex decomposition results for certain subsets of Euclidean spaces were proved before by Breen [2], McKinney [11] and Valentine [21], respectively. A characterization of the sets, which are decomposable into $k$ convex subsets, is given by Lawrence, Hare and Kenell [10] with no restrictions on the set. For example, Valentine [21] proved that the 3-convex closed subsets of the plane $\mathbb{R}^{2}$ are decomposable in unions of three or fewer closed convex sets, i.e., $\gamma(D) \leq 3$. On the other hand, Breen [2] proved that the 3-convex open subsets of the plane are decomposable in unions of four or fewer convex sets, namely, $\gamma(D) \leq 4$. Some other convex decomposition results were proved by Lawrence, Hare and Kenell [10]. More recently, Matoušek and Valtr [14] provided an upper-bound for the convexity number of an $(n+1)$ convex subset of $\mathbb{R}^{d}$ improving a previous upper-bound by Perles-Shelah [15] for $(n+1)$-convex subsets of $\mathbb{R}^{2}$. The convexity number of the complement $\mathbb{R}^{n} \backslash S$ of a finite set $S \subset \mathbb{R}^{n}$ has been extensively treated by Lawrence and Morris [9], where it is expressed as the chromatic number of a certain infinite hypergraph. The first author also provided, in [18], the arguments showing that $\gamma(D)=\aleph_{0}$ whenever $D \subseteq \mathbb{R}^{2}$ is a bounded connected nonconvex and open set with smooth boundary and $\gamma\left(\mathbb{R}^{n} \backslash P\right)=s$, where $P \subseteq \mathbb{R}^{n}$ is an $n$-dimensional polytope with $s$ facets.

The remaining part of the paper is structured as follows. In the next section, we provide a few examples of CIP functions one of which arise by removing the convexity requirement in a global injectivity criterion by Gale and Nikaidô [6]. The rest of the second section is devoted to the open set

$$
\operatorname{Hess}^{+}(f):=\left\{x \in D \mid H_{f}(x) \text { is positive definite }\right\}
$$

associated to some $C^{2}$-smooth regular function $f: D \longrightarrow \mathbb{R}$, where $D \subseteq \mathbb{R}^{n}$ is an open set and $H_{f}(x)$ stands for the Hessian matrix of $f$ at $x \in D$, as the gradient $\nabla f$ and some of its perturbations $\nabla f+A$, are CIP functions, where $A: \mathbb{R}^{n} \longrightarrow \mathbb{R}^{n}$ is a suitable linear operator. Special attention is paid to the polynomial function

$$
f_{a}: \mathbb{R}^{2} \longrightarrow \mathbb{R}, f_{a}(x, y)=\left(x^{2}+y^{2}\right)^{2}-2 a^{2}\left(x^{2}-y^{2}\right),(a>0)
$$

whose zero level set is the famous Bernoulli's lemniscate and the nonempty regular level sets are the famous Cassini's ovals.

In the third section, the CIP quality of the gradient vector field of a $C^{2}$-smooth regular function $f: D \longrightarrow \mathbb{R}$ over the open set $\mathrm{Hess}^{+}(f)$ is exploited to emphasize the convexity number $\gamma\left(\operatorname{Hess}^{+}(f)\right)$ as an upper estimate for the valence of the gradient $\nabla f$ and the perturbed gradient $\nabla f+A$, where $A: \mathbb{R}^{n} \longrightarrow \mathbb{R}^{n}$ is a suitable linear operator.

The last section is devoted to a certain description of the maximal convex subsets of $C \backslash K$, where $C \subseteq \mathbb{R}^{n}$ is a convex open set and $K \subset C$ is a compact set.

\section{EXAMPLES OF CIP FUNCTIONS}

Every global injectivity criterion for functions defined over convex open sets is a potential source of examples of CIP functions on nonconvex sets if we get rid of the convexity assumption in such a global injectivity criterion. Such a criterion for $C^{1}$-smooth maps $F: D \longrightarrow \mathbb{R}^{n}$ on convex open sets $D \subseteq \mathbb{R}^{n}$ is due to Gale-Nikaidô [6] and it ensures the global injectivity of $F$ under the positive definiteness of its Fréchet differentials $(d F)_{x}, x \in D$, namely,

$$
\left\langle(d F)_{x}(y), y\right\rangle>0, \forall x \in D, y \in \mathbb{R}^{n} \backslash\{0\} .
$$


The requirement (2.1) ensures the convex injectivity property of $F: D \longrightarrow \mathbb{R}^{n}$ if we get rid of the convexity requirement on $D$.

For $n=2$, the positive definiteness of the Fréchet differentials $(d F)_{x}, x \in D$ is equivalent to

$$
\operatorname{Re}\left(F_{z}\right)>\left|F_{\bar{z}}\right| \text {, on } D \text {. }
$$

If we increase significantly the regularity of $f$ up to holomorphy, i.e., $F_{\bar{z}} \equiv 0$, then the condition (2.2) is equivalent to

$$
\operatorname{Re}\left(F^{\prime}\right)>0 \text {. }
$$

and it ensures the convex injectivity property of $F$ or the global injectivity when the domain $D$ of $f$ is convex. The latter situation corresponds to the Alexander-Noshiro-Warschawski univalence criterion $[1,17,22]$. If we get rid of the convexity assumption in this injectivity criterion there exists non-injective regular functions, i.e., holomorphic with nonvanishing derivative, satisfying (2.3). Indeed, if the set of deficiency of the domain $D \subseteq \mathbb{C}$ is not totally disconnected, then there are, according to Herzog and Piranian [4] (see also [20]), regular functions $F: D \longrightarrow \mathbb{C}$, which are not injective and $\operatorname{Re}\left(F^{\prime}\right)>0$, but they remain CIP-functions. Recall that the set of deficiency of $D$ is the complement of $D$ relative to the interior of its convex closure. For example, the set of deficiency of $D \backslash K$, where $D \subseteq \mathbb{R}^{n}$ is a convex open set and $K \subseteq D$ is a compact set is precisely $K$. Note that Herzog and Piranian's result do not produce any example of a regular function $F: D \backslash S \longrightarrow \mathbb{C}$, which is not injective and $\operatorname{Re}\left(F^{\prime}\right)>0$, when $K:=S$ is a finite subset of the convex open set $D \subseteq \mathbb{C}$, as the deficiency set $S$ of $D \backslash S$ is totally disconnected. However there are regular non-injective CIP-functions on $D \backslash\{p\}$. For example, when $D:=D^{2}=\{z \in \mathbb{C}|| z \mid<1\}$ is the open unit disk and $p=0$, we have $F: D^{2} \backslash\{0\} \longrightarrow \mathbb{C}, F(z)=z^{2}$ and $1 / F$ are noninjective regular CIP-functions on $D^{2} \backslash\{0\}$. The two regular functions are covering maps over their ranges $D^{2} \backslash\{0\}$ and $\mathbb{C} \backslash \operatorname{cl}\left(D^{2}\right)$, respectively. On the other hand, Herzog and Piranian's result do produce a function on $F: D \backslash P \longrightarrow \mathbb{C}$, where $K:=P \subset D$ is a convex polygon, which is not injective and $\operatorname{Re}\left(F^{\prime}\right)>0$.

The class of $C^{2}$-smooth functions on a certain open set $D \subseteq \mathbb{R}^{n}$ is, through the gradients of the functions in this class, another source of examples of CIP functions. Indeed, the gradient $\nabla f$ of such a function $f: D \longrightarrow \mathbb{R}$ is a CIP function over the open set $\operatorname{Hess}^{+}(f) \subseteq D$, where the hessian matrix $H_{f}$ of $f$ is positive definite, as the positive definiteness of $H_{f}$ at some $x \in O$ is actually condition (2.1) for $\nabla f$ at $x$. Moreover, the perturbation $\nabla f+A$ of the gradient of $f$ with a suitable linear operator $A: \mathbb{R}^{n} \longrightarrow \mathbb{R}^{n}$ remains a CIP function over $\mathrm{Hess}^{+}(f)$. The injectivity of $\nabla f+A$ is ensured, according to [19, Proposition 3.1], by the requirement

$$
\sigma_{f}<\min \left\{b_{A}+\lambda_{A},-\mu_{A}\right\}
$$

where

$$
\sigma_{f}:=\sup _{z \in D}\left\|h_{f}(z)\right\|, b_{A}=\min _{x \in S^{n-1}}\|A x\|
$$

and $\lambda_{A}$ and $\mu_{A}$ denote the smallest and the greatest eigenvalue of the symmetric operator $(A+$ $\left.A^{*}\right) / 2$.

A convex shape of a certain regular sublevel set $f^{-1}((-\infty, c])$ bounded by the regular level set $f^{-1}(c)$ of $f$ completely contained in $\operatorname{Hess}^{+}(f)$ is a reasonable expectation as the positive definiteness of the Hessian matrix over a convex open set ensures the strict convexity of the considered function and the convexity of its sublevel sets as well. In order to simplify the 
notations we shall sometimes omit some parenthesis. In this respect, we may write $f^{-1}(\infty, c]$ instead of $f^{-1}((\infty, c])$.

Example 2.1. We are going to describe the open set $\operatorname{Hess}^{+}\left(f_{a}\right)$ associated to the polynomial function

$$
f_{a}: \mathbb{R}^{2} \longrightarrow \mathbb{R}, f_{a}(x, y)=\left(x^{2}+y^{2}\right)^{2}-2 a^{2}\left(x^{2}-y^{2}\right),(a>0)
$$

and to characterize its level sets $f_{a}^{-1}(c)$ completely contained in $\operatorname{Hess}^{+}\left(f_{a}\right)$ and those bounding convex sublevel sets as well. Recall that the nonempty regular level sets of $f_{a}$ are the famous Cassini's ovals. In this respect, we first observe that all level and sublevel sets of $f_{a}$ are compact as

$$
\lim _{\|(x, y)\| \rightarrow+\infty} f_{a}(x, y)=+\infty .
$$

The zero level set of $f_{a}$, which is critical containing one single critical point $(0,0)$, is the famous Bernoulli's lemniscate. The other critical level is $-a^{4}$ and it is the global minimum of $f_{a}$ attained at the critical points $( \pm a, 0)$. In fact $f_{a}$ is a Morse function with the critical set $(-a, 0),(0,0),(a, 0)$. The minimum points $( \pm a, 0)$ are critical points of index zero and the saddle point $(0,0)$ is the only critical points of index one. The other level sets of $f_{a}$ are all compact and regular and the connected components, of each of them, are diffeomorphic to the circle $S^{1}$, due to the classification theorem of 1-dimensional manifolds. In other words, a regular level set of $f_{a}$ is a disjoint union of finitely many connected closed curves without boundary, each of which is diffeomorphic to the circle $S^{1}$. Consequently, every such closed curve divides the plane $\mathbb{R}^{2}$ in two connected components, due to the Jordan separation Theorem. One of these components (the interior) is bounded and the other one (the exterior) is unbounded and the closed curve is their common boundary. In this particular case the regular levels $f_{a}^{-1}(c)$, i.e. Cassini's ovals, of $f_{a}$ are either disjoint unions of two closed connected curves (for $c \in\left(-a^{4}, 0\right)$ ) and one single closed connected curve (for $c>0$ ).

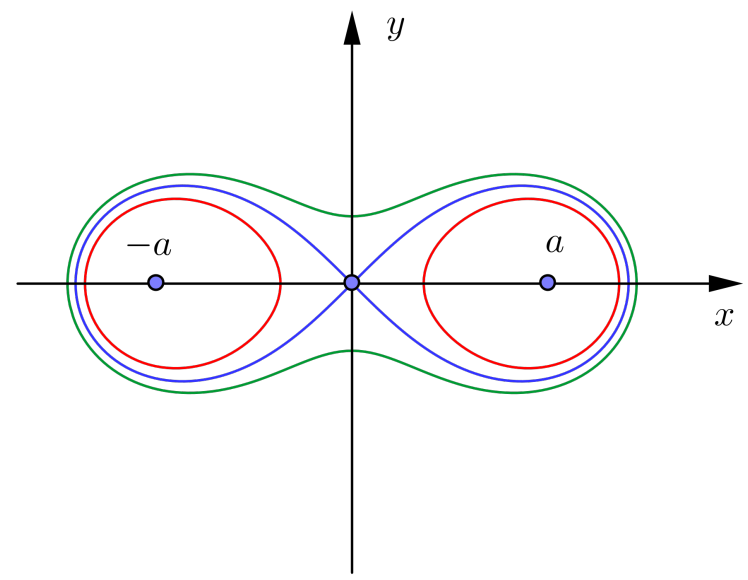

The critical level $f_{a}^{-1}(0)$, a negative regular level and a positive regular level of $f_{a}$ 
In what follows, we shall first characterize the regular level sets $f_{a}^{-1}(c)$ bounding convex sublevel sets. By combining [3, Proposition 1, p. 397] with [8, Formula 3.7] one can show that every connected component of $f_{a}^{-1}(-\infty, c]$ is convex by showing that each component of its boundary $f_{a}^{-1}(c)$ is a closed simple convex curve. In this respect, it remains only to characterize those regular values $c$ of $f_{a}$ for which the determinant function

$$
\left|\begin{array}{lll}
\left(f_{a}\right)_{x x} & \left(f_{a}\right)_{x y} & \left(f_{a}\right)_{x} \\
\left(f_{a}\right)_{y x} & \left(f_{a}\right)_{y y} & \left(f_{a}\right)_{y} \\
\left(f_{a}\right)_{x} & \left(f_{a}\right)_{y} & 0
\end{array}\right|
$$

has constant sign over the compact nonempty regular level set

$$
f_{a}^{-1}(c):\left(x^{2}+y^{2}\right)^{2}-2 a^{2}\left(x^{2}-y^{2}\right)=c \text { (Cassini's oval). }
$$

A fairly simple calculation shows that the restriction of the determinant function (2.5) to $f_{a}^{-1}(c)$ is, up to a multiplicative constant, equal to

$$
3\left(x^{2}+y^{2}\right)^{2}-c .
$$

The minimum and maximum value of determinant function (2.5) over the Cassini's oval $f_{a}^{-1}(c)$ can be found by means of the Lagrange multipliers. The associated Lagrange function is

$$
\mathscr{L}(x, y, \lambda)=\left(x^{2}+y^{2}\right)^{2}-\lambda\left(\left(x^{2}+y^{2}\right)^{2}-2 a^{2}\left(x^{2}-y^{2}\right)-c\right)
$$

and

$$
\begin{aligned}
\nabla \mathscr{L}_{(x, y, \lambda)}=0 & \Longleftrightarrow\left\{\begin{array}{l}
4 x\left(x^{2}+y^{2}\right)-4 \lambda x\left(x^{2}+y^{2}\right)+4 a^{2} \lambda x=0, \\
4 y\left(x^{2}+y^{2}\right)-4 \lambda y\left(x^{2}+y^{2}\right)-4 a^{2} \lambda y=0, \\
\left(x^{2}+y^{2}\right)^{2}-2 a^{2}\left(x^{2}-y^{2}\right)=c,
\end{array}\right. \\
& \stackrel{c \neq 0}{\Longleftrightarrow}\left\{\begin{array}{l}
x=0, \\
y^{2}-\lambda y^{2}-a^{2} \lambda=0, \\
y^{4}+2 a^{2} y^{2}-c=0,
\end{array}\right. \\
& \text { or }\left\{\begin{array}{l}
x^{2}-\lambda x^{2}+a^{2} \lambda=0, \\
y=0, \\
x^{4}-2 a^{2} x^{2}-c=0, \\
x^{2}+y^{2}-\lambda\left(x^{2}+y^{2}\right)+a^{2} \lambda=0, \\
x^{2}+y^{2}-\lambda\left(x^{2}+y^{2}\right)-a^{2} \lambda=0, \\
\left(x^{2}+y^{2}\right)^{2}-2 a^{2}\left(x^{2}-y^{2}\right)=c .
\end{array}\right.
\end{aligned}
$$

While the last system is inconsistent, the first two systems admit the solutions

$$
\begin{gathered}
\left(0, \pm \sqrt{-a^{2}+\sqrt{a^{4}+c}}, \frac{\sqrt{-a^{2}+\sqrt{a^{4}+c}}}{\sqrt{a^{4}+c}}\right), \\
\left( \pm \sqrt{a^{2}+\sqrt{a^{4}+c}}, 0, \frac{\sqrt{a^{2}+\sqrt{a^{4}+c}}}{\sqrt{a^{4}+c}}\right)
\end{gathered}
$$

for $c>0$ and

$$
\begin{gathered}
\left( \pm \sqrt{a^{2}-\sqrt{a^{4}+c}}, 0,-\frac{\sqrt{a^{2}-\sqrt{a^{4}+c}}}{\sqrt{a^{4}+c}}\right), \\
\left( \pm \sqrt{a^{2}+\sqrt{a^{4}+c}}, 0, \frac{\sqrt{a^{2}+\sqrt{a^{4}+c}}}{\sqrt{a^{4}+c}}\right)
\end{gathered}
$$


for $c<0$. The value of the polynomial function $3\left(x^{2}+y^{2}\right)^{2}-c$ on the points

$$
\left(0, \pm \sqrt{-a^{2}+\sqrt{a^{4}+c}}\right)
$$

is $6 a^{4}-6 a^{2} \sqrt{a^{4}+c}+2 c$, and this is the minimum value of $3\left(x^{2}+y^{2}\right)^{2}-c$ over $f_{a}^{-1}(c)$. Also, its value on the points $\left( \pm \sqrt{a^{2}-\sqrt{a^{4}+c}}, 0\right)$ is $6 a^{4}+6 a^{2} \sqrt{a^{4}+c}+2 c$, and this is the maximum of the function $3\left(x^{2}+y^{2}\right)^{2}-c$ over $f_{a}^{-1}(c)$. Thus, the polynomial function $3\left(x^{2}+y^{2}\right)^{2}-c$, alongside with the determinant function (2.5) does not change the sign if and only if

$$
6 a^{4}-6 a^{2} \sqrt{a^{4}+c}+2 c \geq 0,
$$

as $6 a^{4}+6 a^{2} \sqrt{a^{4}+c}+2 c>0$. The inequality (2.7) holds if and only if

$$
c^{2}-3 a^{4} c>0 \text {, i.e. } c \in(-\infty, 0] \cup\left[3 a^{4},+\infty\right) \text {. }
$$

We need to exclude the critical values zero and $-a^{4}$, as the Bernoulli's lemniscate has a singularity and the level set $f_{a}^{-1}\left(-a^{4}\right)$ consists in two points, for which the curvature is not defined. In other words, the level set $f_{a}^{-1}(c)$ is regular, nonempty and its curvature does not change the sign if and only if $c \in\left(-a^{4}, 0\right) \cup\left[3 a^{4},+\infty\right)$. In fact, every sublevel set $f_{a}^{-1}(-\infty, c]$, for $c \in\left(-a^{4}, 0\right)$, has two compact convex components and is bounded by the regular level $f_{a}^{-1}(c)$, which is the union of two Cassini's ovals. On the other hand, the regular value $3 a^{4}$ of $f_{a}$ is the first one for which the sublevel set $f_{a}^{-1}\left(-\infty, 3 a^{4}\right]$ is compact, convex and bounded by one single Cassini's oval $f_{a}^{-1}\left(3 a^{4}\right)$, which is a connected convex regular curve. Indeed, for every regular value $c$ of $f_{a}$, greater than $3 a^{4}$, the sublevel set $f_{a}^{-1}(-\infty, c]$ is compact, convex and bounded by one single Cassini's oval $f_{a}^{-1}(c)$, which is a connected convex regular curve.

Remark 2.1. Similar arguments show that the level set $g_{a}^{-1}(c)$ of the polynomial function

$$
g_{a}: \mathbb{R}^{2} \longrightarrow \mathbb{R}, g_{a}(x, y)=\left(x^{2}+y^{2}\right)^{2}+2 a^{2}\left(x^{2}-y^{2}\right)
$$

is regular, nonempty and its curvature does not change the sign if and only if $c \in\left(-a^{4}, 0\right) \cup$ $\left[3 a^{4},+\infty\right)$. In fact $g_{a}=f_{a} \circ r_{b}$, where

$$
r_{b}: \mathbb{R}^{2} \longrightarrow \mathbb{R}^{2}, r_{b}(x, y)=(y, x)
$$

is the reflection in the first bisector $b: x-y=0$. Thus, the following sets associated to the functions $f_{a}$ and $g_{a}$ are symmetric with respect to the first bisector:

(1) the critical sets $C\left(f_{a}\right)$ and $C\left(g_{a}\right)$ of the functions $f_{a}$ and $g_{a}$;

(2) the level sets $f_{a}^{-1}(c)$ and $g_{a}^{-1}(c)$, for all $c \in \mathbb{R}$. In particular, the two level sets are simultaneously empty;

(3) the sublevel sets $f_{a}^{-1}(-\infty, c]$ and $g_{a}^{-1}(-\infty, c]$, for all $c \in \mathbb{R}$.

The last objective of Example (2.1) was to characterize the level sets $f_{a}^{-1}(c)$ of $f_{a}$ completely contained in $\operatorname{Hess}^{+}\left(f_{a}\right)$. In this respect, we first need to find $\mathrm{Hess}^{+}\left(f_{a}\right)$. The Hessian matrix of $f_{a}$ is

$$
H_{f_{a}}(x, y)=\left[\begin{array}{cc}
12 x^{2}+4 y^{2}-4 a^{2} & 8 x y \\
8 x y & 4 x^{2}+12 y^{2}+4 a^{2}
\end{array}\right] .
$$

Recall that $H_{f_{a}}(x, y)$ is positive definite if and only if its eigenvalues

$$
\lambda_{1,2}=8\left(x^{2}+y^{2}\right) \pm 4 \sqrt{f_{a}(x, y)+a^{4}}
$$


are positive [5, Theorem 7.2.1], i.e.,

$$
\operatorname{Hess}^{+}\left(f_{a}\right)=\left\{(x, y) \in \mathbb{R}^{2} \mid 3\left(x^{2}+y^{2}\right)^{2}+2 a^{2}\left(x^{2}-y^{2}\right)>a^{4}\right\}=g_{\frac{a}{\sqrt{3}}}^{-1}\left(\frac{a^{4}}{3},+\infty\right) \text {. }
$$

The boundary of $\mathrm{Hess}^{+}\left(f_{a}\right)$ is the compact connected smooth regular curve

$$
\left\{(x, y) \in \mathbb{R}^{2} \mid 3\left(x^{2}+y^{2}\right)^{2}+2 a^{2}\left(x^{2}-y^{2}\right)=a^{4}\right\}=g_{\frac{a}{\sqrt{3}}}^{-1}\left(\frac{a^{4}}{3}\right)
$$

and the open set $\operatorname{Hess}^{+}\left(f_{a}\right)$ is the unbounded connected component of

$$
\mathbb{R}^{2} \backslash \partial \operatorname{Hess}^{+}\left(f_{a}\right)=\mathbb{R}^{2} \backslash g_{\frac{a}{\sqrt{3}}}^{-1}\left(\frac{a^{4}}{3}\right)
$$

which is therefore nonconvex.

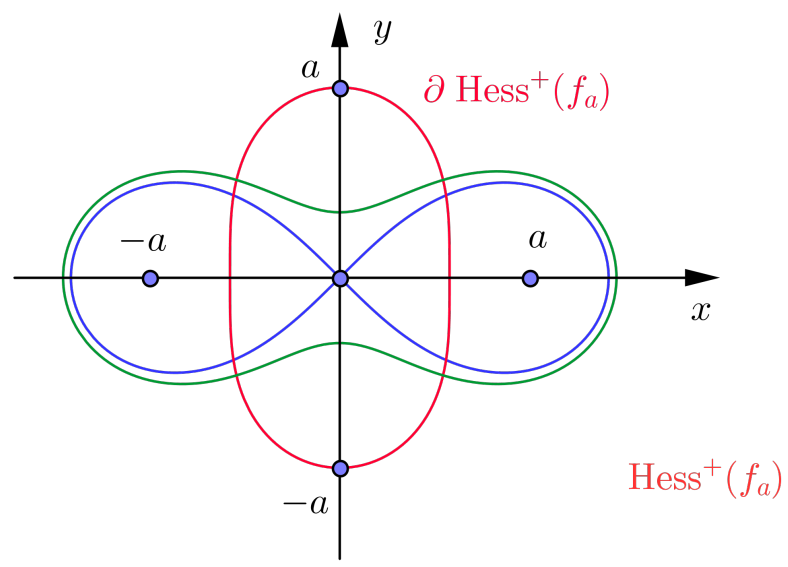

The boundary $\partial \operatorname{Hess}^{+}\left(f_{a}\right)$,

the critical level $\mathrm{f}_{\mathrm{a}}^{-1}(0)$ and a positive regular value of $f_{a}$

FigURE 2.

The maximum and minimum points of $f_{a}$ subject to the constraint $\partial \operatorname{Hess}^{+}\left(f_{a}\right): g_{\frac{a}{\sqrt{3}}}(x, y)=$ $\frac{a^{4}}{3}$, as well as their values through $f_{a}$, provide essential information about the level sets of $f_{a}$ completely contained in $\mathrm{Hess}^{+}\left(f_{a}\right)$. The connected components of these level sets happen to bound convex sets and sometimes they are connected as well. In order to justify these observations, we first determine the maximum and minimum points of $f_{a}$ subject to the constraint $\partial \operatorname{Hess}^{+}\left(f_{a}\right): g_{\frac{a}{\sqrt{3}}}(x, y)=\frac{a^{4}}{3}$, which is equivalent to $3\left(x^{2}+y^{2}\right)^{2}+2 a^{2}\left(x^{2}-y^{2}\right)=a^{4}$. The associate Lagrange function is

$$
\begin{aligned}
\mathscr{L}(x, y, \lambda) & =f_{a}(x, y)-\lambda\left(3\left(x^{2}+y^{2}\right)^{2}+2 a^{2}\left(x^{2}-y^{2}\right)-a^{4}\right) \\
& =\left(x^{2}+y^{2}\right)^{2}-2 a^{2}\left(x^{2}-y^{2}\right)-\lambda\left(3\left(x^{2}+y^{2}\right)^{2}+2 a^{2}\left(x^{2}-y^{2}\right)-a^{4}\right)
\end{aligned}
$$


and its gradient $\nabla \mathscr{L}_{(x, y, \lambda)}=\left(\mathscr{L}_{x}, \mathscr{L}_{y}, \mathscr{L}_{\lambda}\right)$ vanishes if and only if

$$
\nabla \mathscr{L}(x, y, \lambda)=0 \Leftrightarrow\left\{\begin{array}{l}
4 x\left((1-3 \lambda)\left(x^{2}+y^{2}\right)-a^{2}(1+\lambda)\right)=0, \\
4 y\left((1-3 \lambda)\left(x^{2}+y^{2}\right)+a^{2}(1+\lambda)\right)=0 \\
3\left(x^{2}+y^{2}\right)^{2}+2 a^{2}\left(x^{2}-y^{2}\right)=a^{4}
\end{array}\right.
$$

i.e.,

$$
\begin{aligned}
& \left\{\begin{array} { l } 
{ x = 0 } \\
{ ( 1 - 3 \lambda ) y ^ { 2 } + a ^ { 2 } ( 1 + \lambda ) = 0 , } \\
{ 3 y ^ { 4 } - 2 a ^ { 2 } y ^ { 2 } - a ^ { 4 } = 0 , }
\end{array} \text { or } \left\{\begin{array}{l}
y=0, \\
(1-3 \lambda) x^{2}-a^{2}(1+\lambda)=0, \\
3 x^{4}+2 a^{2} x^{2}-a^{4}=0,
\end{array}\right.\right. \\
& \text { or } \begin{array}{l}
(1-3 \lambda)\left(x^{2}+y^{2}\right)-a^{2}(1+\lambda)=0, \\
(1-3 \lambda)\left(x^{2}+y^{2}\right)+a^{2}(1+\lambda)=0, \\
3\left(x^{2}+y^{2}\right)^{2}+2 a^{2}\left(x^{2}-y^{2}\right)=a^{4},
\end{array}
\end{aligned}
$$

While the first and second system have the solutions $(0, \pm a, 1)$ and $\left( \pm \frac{a}{\sqrt{3}}, 0,-\frac{1}{3}\right)$ respectively, the last one is inconsistent. One can easily see that $(0, \pm a)$ and $\pm \frac{a}{\sqrt{3}}$ are the constrained maximum and minimum points of $f_{a}$ respectively with respect to the constraint

$$
3\left(x^{2}+y^{2}\right)^{2}+2 a^{2}\left(x^{2}-y^{2}\right)=a^{4} .
$$

The constrained maximum and minimum of $f_{a}$ are $f_{a}( \pm a, 0)=3 a^{4}$ and $f_{a}\left( \pm \frac{a}{\sqrt{3}}, 0\right)=-\frac{5 a^{4}}{9}$, respectively. Note that these data remain valid, not only for the constraint $\partial \operatorname{Hess}^{+}\left(f_{a}\right)=$ $g_{\frac{a}{\sqrt{3}}}^{-1}\left(\frac{a^{4}}{3}\right)$, but also for

$$
\begin{aligned}
\mathbb{R}^{2} \backslash \operatorname{Hess}^{+}\left(f_{a}\right) & =g_{\frac{a}{\sqrt{3}}}^{-1}\left(-\infty, \frac{a^{4}}{3}\right]=g_{\frac{a}{\sqrt{3}}}^{-1}\left(-\infty, \frac{a^{4}}{3}\right) \cup g_{\frac{a}{\sqrt{3}}}^{-1}\left(\frac{a^{4}}{3}\right) \\
& =g_{\frac{a}{\sqrt{3}}}^{-1}\left(-\infty, \frac{a^{4}}{3}\right) \cup \partial \operatorname{Hess}^{+}\left(f_{a}\right),
\end{aligned}
$$

as the only critical point of $f_{a}$ in $g_{\frac{a}{\sqrt{3}}}\left(-\infty, \frac{a^{4}}{3}\right)$ is $(0,0)$, which is a saddle point of $f_{a}$, not a local minimum or a local maximum. More precisely,

$$
(0, \pm a) \text { and }\left( \pm \frac{a}{\sqrt{3}}, 0\right)
$$

are still the constrained maximum and minimum points of $f_{a}$, respectively, with respect to constraint $g_{\frac{a}{\sqrt{3}}}(x, y) \leq \frac{a^{4}}{3}$. The constrained maximum and minimum of $f_{a}$, with respect to the constraint $g_{\frac{a}{\sqrt{3}}}(x, y) \leq \frac{a^{4}}{3}$, are still $f_{a}( \pm a, 0)=3 a^{4}$ and $f_{a}\left( \pm \frac{a}{\sqrt{3}}, 0\right)=-\frac{5 a^{4}}{9}$, respectively. This particularly shows that

$$
f_{a}\left(g_{\frac{a}{\sqrt{3}}}^{-1}\left(\left(-\infty, \frac{a^{4}}{3}\right]\right)\right)=\left[-\frac{5 a^{4}}{9}, 3 a^{4}\right]
$$


as the set $g_{\frac{a}{\sqrt{3}}}^{-1}\left(\left(-\infty, \frac{a^{4}}{3}\right]\right)$ is convex. Therefore

$$
f_{a}^{-1}(c) \subseteq g_{\frac{a}{\sqrt{3}}}^{-1}\left(\left[\frac{a^{4}}{3},+\infty\right)\right)=\operatorname{clHess}^{+}\left(f_{a}\right)
$$

whenever

$$
c \in\left[-a^{4},-\frac{5 a^{4}}{9}\right] \cup\left[3 a^{4},+\infty\right) .
$$

Conversely, if $f_{a}^{-1}(c) \subseteq g_{\frac{a}{\sqrt{3}}}^{-1}\left(\left(a^{4},+\infty\right]\right)=\operatorname{cl~Hess}^{+}\left(f_{a}\right)$, then

$$
c \in\left[-a^{4},-\frac{5 a^{4}}{9}\right] \cup\left[3 a^{4},+\infty\right),
$$

or, equivalently, $f_{a}^{-1}(c) \nsubseteq g_{\frac{a}{\sqrt{3}}}^{-1}\left(\left[\frac{a^{4}}{3},+\infty\right)\right)=\mathrm{cl} \mathrm{Hess}^{+}\left(f_{a}\right)$ for $c \in\left(-\frac{5 a^{4}}{9}, 3 a^{4}\right)$. Indeed, if $c \in\left[-a^{4}, 0\right]$, then one can easily show that

$$
\begin{aligned}
\left( \pm \sqrt{a^{2}-\sqrt{a^{4}+c}}, 0\right) & \in f_{a}^{-1}(c) \backslash g_{\frac{a}{\sqrt{3}}}^{-1}\left(\left[\frac{a^{4}}{3},+\infty\right)\right) \\
& =f_{a}^{-1}(c) \cap g_{\frac{a}{\sqrt{3}}}^{-1}\left(\left(-\infty, \frac{a^{4}}{3}\right)\right)
\end{aligned}
$$

and for $c \in\left(0,3 a^{4}\right)$, then one can easily show that

$$
\begin{aligned}
\left(0, \pm \sqrt{-a^{2}+\sqrt{a^{4}+c}}\right) & \in f_{a}^{-1}(c) \backslash g_{\frac{a}{\sqrt{3}}}^{-1}\left(\left[\frac{a^{4}}{3},+\infty\right)\right) \\
& =f_{a}^{-1}(c) \cap g_{\frac{a}{\sqrt{3}}}^{-1}\left(\left(-\infty, \frac{a^{4}}{3}\right)\right) .
\end{aligned}
$$

Note that $\partial \operatorname{Hess}^{+}\left(f_{a}\right)=g_{\frac{a}{\sqrt{3}}}^{-1}\left(\frac{a^{4}}{3}\right)$ is the first positive regular level set of $g_{\frac{a}{\sqrt{3}}}$, which is convex

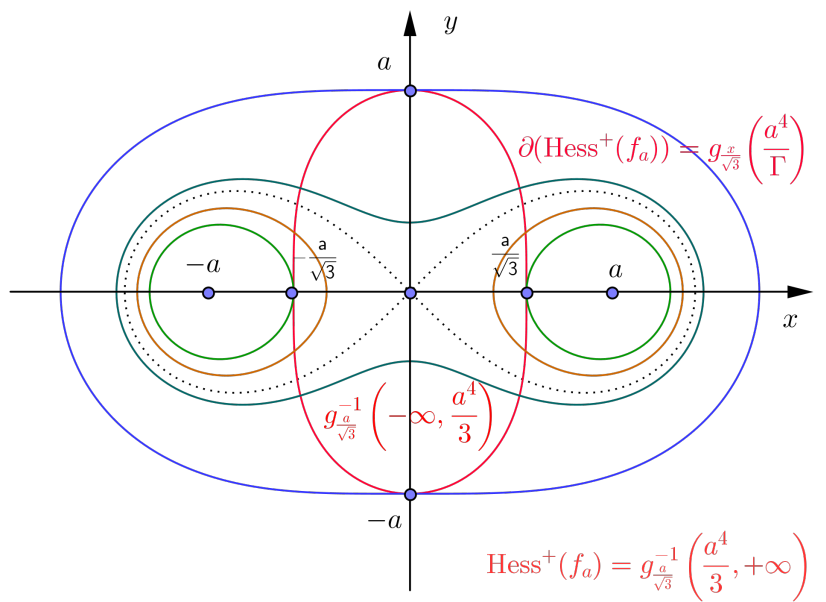

The boundary $\partial\left(\operatorname{Hess}^{+}\left(\mathrm{f}_{\mathrm{a}}\right)\right)$, the last negative regular level and the first positive convex regular level of $f_{a}$ contained in $\mathrm{Cl} \mathrm{Hess}^{+}\left(\mathrm{f}_{\mathrm{a}}\right)$. Positive nonconvex regular level and disconnected negative regular level of $f_{a}$ with convex components, which are not contained in $\mathrm{Cl} \mathrm{Hess}^{+}\left(f_{a}\right)$.

FIGURE 3. 
and $f_{a}^{-1}\left(3 a^{4}\right)$ is the first positive regular level of $f_{a}$, which is convex.

\section{AN UPPER BOUND FOR THE VALENCE OF SOME GRADIENTS AND PERTURBED GRADIENTS IN TERMS OF $\gamma$}

As we have already mentioned, the valence of any CIP-function $F: D \longrightarrow \mathbb{R}^{n}$ cannot exceed any number of convex subsets of $D$ needed to cover $D$. In other words, if $F: D \longrightarrow \mathbb{R}^{n}$ is a CIP-function, then

$$
\operatorname{Val}(F) \leq \gamma(D)
$$

where $\operatorname{Val}(F):=\sup \left\{\operatorname{card} F^{-1}(y): y \in \mathbb{R}^{n}\right\}$ stands for the valence of $F$, as defined in [16]. In particular,

$$
\operatorname{Val}\left(\left.\nabla f\right|_{\mathrm{Hess}^{+}(f)}\right) \leq \gamma\left(\operatorname{Hess}^{+}(f)\right)
$$

where $f: D \longrightarrow \mathbb{R}$ is a $C^{2}$-smooth function on the open set $D \subseteq \mathbb{R}^{n}$ such that $\operatorname{Hess}^{+}(f) \neq \emptyset$. Moreover

$$
\left.\operatorname{Val}(\nabla f+A)\right|_{\operatorname{Hess}^{+}(f)} \leq \gamma\left(\operatorname{Hess}^{+}(f)\right),
$$

where $A: \mathbb{R}^{n} \longrightarrow \mathbb{R}^{n}$ is a suitable linear operator. The upper bounds 3.2 and 3.3 for the valences $\operatorname{Val}\left(\left.\nabla f\right|_{\mathrm{Hess}^{+}(f)}\right)$ and $\left.\operatorname{Val}(\nabla f+A)\right|_{\mathrm{Hess}^{+}(f)}$ respectively are obvious when $n=2$ and the boundary of $\mathrm{Hess}^{+}(f)$ is a smooth curve or $\mathrm{Hess}^{+}(f)$ is a convex open set. If Hess ${ }^{+}(f)$ is nonconvex and its boundary a smooth curve, then

$$
\gamma\left(\operatorname{Hess}^{+}(f)\right)=\boldsymbol{\aleph}_{0}
$$

(see [18, Theorem 2.4]). However these type of upper bounds are interesting for the restrictions of $\nabla f$ and $\nabla f+A$ to every open subset $\mathscr{P}$ of $\operatorname{Hess}^{+}(f)$ with polyhedral boundary, as the convexity number associated to such a subset is finite.

If $\mathscr{P} \subseteq \operatorname{Hess}^{+}(f)$ happens to be $\mathbb{R}^{n} \backslash P$, where $P \subseteq \mathbb{R}^{n}$ is an $n$-dimensional polytope with $s$ facets, then

$$
\begin{gathered}
\operatorname{Val}\left(\left.\nabla f\right|_{\mathbb{R}^{n} \backslash P}\right) \leq \gamma\left(\mathbb{R}^{n} \backslash P\right)=s, \\
\left.\operatorname{Val}(\nabla f+A)\right|_{\mathbb{R}^{n} \backslash P} \leq \gamma\left(\mathbb{R}^{n} \backslash P\right)=s .
\end{gathered}
$$

Example 3.1. We are going to use the estimates (3.4) and (3.5) adjusted to some open subsets of $\operatorname{Hess}^{+}\left(f_{a}\right)$ with polygonal boundary, where

$$
f_{a}: \mathbb{R}^{2} \longrightarrow \mathbb{R}, f_{a}(x, y)=\left(x^{2}+y^{2}\right)^{2}-2 a^{2}\left(x^{2}-y^{2}\right) .
$$

In this respect, we inscribe $\partial \operatorname{Hess}^{+}\left(f_{a}\right)$ in a polygon $P$ that has $s$ edges and observe that

$$
\operatorname{Val}\left(\left.\nabla f_{a}\right|_{\mathbb{R}^{2} \backslash P}\right) \leq s=\gamma\left(\mathbb{R}^{2} \backslash P\right) .
$$

On the other hand, we may choose $P$ so that the critical points $( \pm a, 0)$ of $f_{a}$ are in $\mathbb{R}^{2} \backslash P$. This choice ensures that

$$
\operatorname{Val}\left(\left.\nabla f_{a}\right|_{\mathbb{R}^{2} \backslash P}\right) \geq 2 \text {. }
$$

This lower estimate for $\operatorname{Val}\left(\left.\nabla f_{a}\right|_{\mathbb{R}^{2} \backslash P}\right)$ is strengthened by the fact that the gradient $\nabla f_{a}$ evaluated at intersection point

$$
\left( \pm \frac{\sqrt{3 a^{4}-c}}{2 a}, \frac{\sqrt{a^{4}+c}}{2 a}\right)
$$


of the circle $x^{2}+y^{2}=a^{2}$ with the level set $f_{a}^{-1}(c)$ is $\left(0,4 a \sqrt{a^{4}+c}\right)$, for every $-a^{4} \leq c<3 a^{4}$. Therefore, we have the estimates

$$
2 \leq \operatorname{Val}\left(\left.\nabla f_{a}\right|_{\mathbb{R}^{2} \backslash P}\right) \leq s,
$$

whenever the relation

$$
\left( \pm \frac{\sqrt{3 a^{4}-c}}{2 a}, \frac{\sqrt{a^{4}+c}}{2 a}\right) \in \mathbb{R}^{2} \backslash P
$$

holds for some $c \in\left[-a^{4}, 3 a^{4}\right)$. If we choose $P$ to be a triangle $T$, then we obtain, under the same

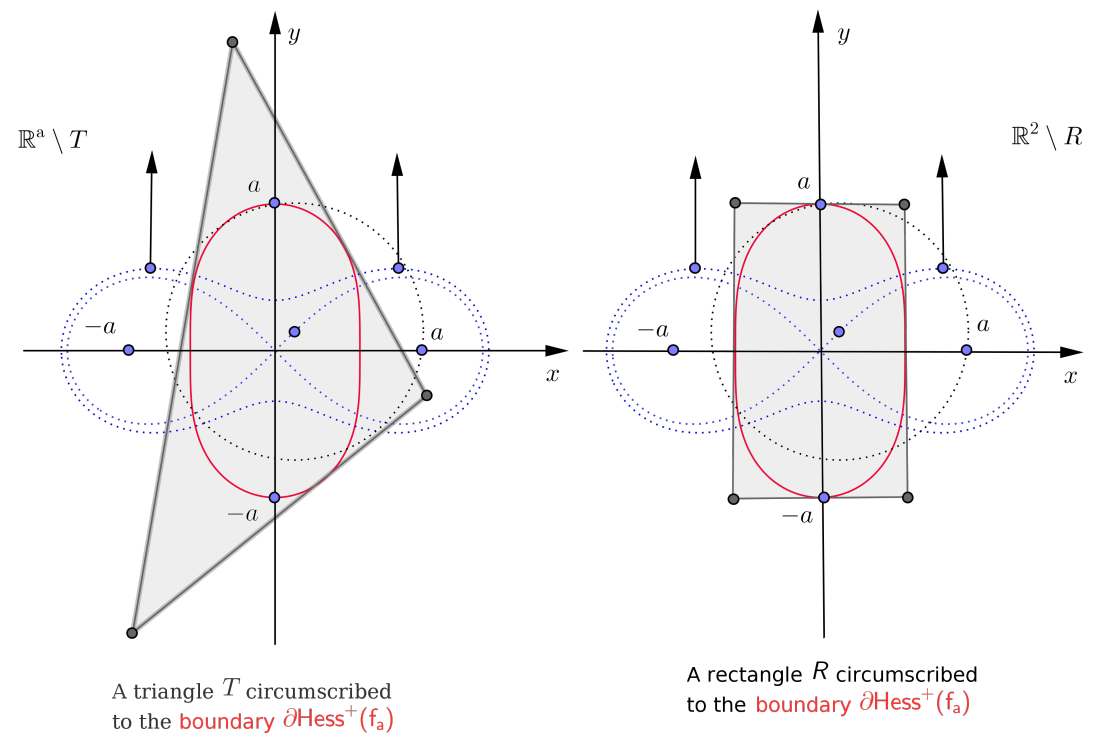

FIGURE 4.

circumstances, the following estimates

$$
2 \leq \operatorname{Val}\left(\left.\nabla f_{a}\right|_{\mathbb{R}^{2} \backslash P}\right) \leq 3 .
$$

Note that the computations that refer to $\operatorname{Val}\left(\left.\nabla f_{a}\right|_{\mathbb{R}^{2} \backslash P}\right)$ are also true if the gradient of $f_{a}$ is perturbed with a suitable linear operator. More precisely,

$$
\left.\operatorname{Val}\left(\nabla f_{a}+A\right)\right|_{\mathbb{R}^{2} \backslash P} \leq s,
$$

where $P$ is a polygon with $s$ edges such that $\mathbb{R}^{2} \backslash \operatorname{Hess}^{+}\left(f_{a}\right) \subseteq P$ and $A: \mathbb{R}^{2} \rightarrow \mathbb{R}^{2}$ is a linear operator with specific properties. For example, $A$ might be $R_{\theta}-i_{D}$, where $R_{\theta}$ is the rotation through an angle $\theta \in(0, \pi / 2)$ and $D$ is an open subset of $\operatorname{Hess}^{+}\left(f_{a}\right)$, with polygonal boundary, subject to the extra constraint $\Delta f_{a} \leq \operatorname{det}\left(H_{f_{a}}\right)$ and $i_{D}: D \hookrightarrow \mathbb{R}^{2}$ is the inclusion map (see [12, Theorem 1.3]).

\section{MaXimal CONVEX SUBSETS OF CONVEX OPEN SETS WITH COMPACT CONVEX SETS REMOVED}

Since every convex subset of a given open set $D \subseteq \mathbb{R}^{n}$ is contained in a maximal convex subset of $D$, it follows that $\gamma(D)$ can be equally described as the smallest possible number 
$k$ with the property that $D$ can be covered by $k$ maximal convex subsets of $D$. Recall that the maximal convex subsets of $\mathbb{R}^{n}$ with convex sets removed were characterized before by Martínez-Legaz and Singer [13] as being the convex subsets of $\mathbb{R}^{n}$, shortly called hemispaces, whose complements are convex too. A convex decomposition of $D$ by $\gamma(D)$ convex subsets of $D$ is said to be minimal. For instance, every minimal convex decomposition of a punctured convex open set has maximal convex components. Examples of convex decompositions, of a convex open set with a finite number of points (or a polytope) removed, are provided by [18] and the convex components of these decompositions are usually maximal.

It is therefore worth to provide alternative descriptions of the maximal convex subsets of $C \backslash K$ by means, for instance, of linear functionals $\varphi: \mathbb{R}^{n} \longrightarrow \mathbb{R}$. We rely on the representation of every open subset of the real line as a countable union of open intervals, which are basically the maximal open intervals contained in that set. We shall apply this fact to the open subset $\mathbb{R} \backslash \varphi(K)$.

Theorem 4.1. Let $C$ be a convex open subset of $\mathbb{R}^{n}$ and $K$ be a nonempty compact subset of $C$. If $\varphi: \mathbb{R}^{n} \longrightarrow \mathbb{R}$ is a linear functional, then

$$
\varphi^{-1}(I) \cap(C \backslash K)
$$

is maximal, among the convex open subsets of $C \backslash K$, where I is some maximal open subinterval of the open set $\mathbb{R} \backslash \varphi(K)$.

Proof. Since every linear functional $\varphi: \mathbb{R}^{n} \longrightarrow \mathbb{R}$ is a continuous function and $K$ is a compact subset of $\mathbb{R}^{n}$, it follows that $\varphi(K)$ is a compact subset of the real line. Thus $\mathbb{R} \backslash \varphi(K)$ is a union of countably many open intervals $\left\{I_{j}\right\}$, each of which a maximal open subinterval of $\mathbb{R} \backslash \varphi(K)$, as $\mathbb{R} \backslash \varphi(K)$ is an open subset of the real line. Note that either $\inf I \in \mathbb{R}$ or $\sup I \in \mathbb{R}$ for every maximal open subinterval $I$ of $\mathbb{R} \backslash \varphi(K)$, as every such $I$ is a proper subinterval of $\mathbb{R}$. Moreover $\varphi^{-1}(\inf I) \cap \partial K \neq \emptyset$ and/or $\varphi^{-1}(\sup I) \cap \partial K \neq \emptyset$ whenever inf $I \in \mathbb{R}$ and/or $\sup I \in \mathbb{R}$ respectively. Assume that

$$
\begin{array}{r}
\inf I \in \mathbb{R} \text { and } \\
\varphi^{-1}(\inf I) \cap \partial K=\emptyset .
\end{array}
$$

In such a case, we would have $(\inf I-\varepsilon, \sup I) \subseteq \mathbb{R} \backslash \varphi(K)$ for $\varepsilon>0$ small enough, as otherwise (inf $I-\frac{1}{n}$, inf $\left.I\right] \cap \varphi(K) \neq \emptyset$, for every $n \geq 1$. In other words, for each $n \geq 1$, there exists some $x_{n} \in K$ such that $\varphi\left(x_{n}\right) \in\left(\inf I-\frac{1}{n}, \inf I\right]$ and therefore $\varphi\left(x_{n}\right) \longrightarrow \inf I$. Since $K$ is compact, the sequence $\left(x_{n}\right)$ has a subsequence, still denoted by $\left(x_{n}\right)$, converging to some limit $x \in K$. Thus

$$
\varphi(K) \ni \varphi(x)=\inf I \in \operatorname{cl}(\mathbb{R} \backslash \varphi(K)),
$$

which shows that $\varphi(x)=\inf I \in \partial(\varphi(K))$, and therefore $x \in \partial K$ as the relation $x \in \operatorname{int}(K)$ would imply $\varphi(x) \in \varphi(\operatorname{int}(K)) \subseteq \operatorname{int}(\varphi(K))$, due to the openness of $\varphi$. We just showed that $x \in \varphi^{-1}(\inf I) \cap \partial K$ a contradiction with the assumption (4.1). The convexity of $\varphi^{-1}(I) \cap(C \backslash K)$ follows from the equality $\varphi^{-1}(I) \cap(C \backslash K)=\varphi^{-1}(I) \cap C$ and the convexities of $\varphi^{-1}(I)$ and $C$. While the inclusion $\varphi^{-1}(I) \cap(C \backslash K) \subseteq \varphi^{-1}(I) \cap C$ is obvious, for the opposite one we only need to observe that $\varphi^{-1}(I) \cap C \cap K \subseteq \varphi^{-1}(I) \cap K \subseteq \varphi^{-1}(\mathbb{R} \backslash \varphi(K)) \cap K=\emptyset$. Assume that $D$ is a convex open subset of $C \backslash K$ such that $\varphi^{-1}(I) \cap(C \backslash K) \subseteq D$ for some maximal open subinterval $I$ of the open set $\mathbb{R} \backslash \varphi(K)$ and $D \backslash\left[\varphi^{-1}(I) \cap(C \backslash K)\right] \neq \emptyset$. If $\inf I \in \mathbb{R}$ and $\sup I=\infty$, then

$$
\varphi(y) \leq \inf I \text { for every } y \in O \backslash\left[\varphi^{-1}(I) \cap(C \backslash K)\right],
$$


as otherwise every such $y$ would belong to $\varphi^{-1}(I) \cap(C \backslash K)$. If the inequality (4.2) is strict, i.e., $\varphi(y)<\inf I$ and $y_{0} \in \varphi^{-1}(\inf I) \cap \partial K$, then

$$
z_{0}:=y+t_{0}\left(y_{0}-y\right) \in \varphi^{-1}(I) \cap C=\varphi^{-1}(I) \cap(C \backslash K) \subset O
$$

for some $t_{0}>1$, as $C$ is open and the inequality $\varphi\left(z_{0}\right)>\inf I$ is equivalent to $\left(t_{0}-1\right)(\inf I-\varphi(y))$ $>0$. Thus $y_{0}=y+t_{0}^{-1}\left(z_{0}-y\right)$ would belong to $D \subseteq C \backslash K$, a contradiction with the initial choice of $y_{0} \in \varphi^{-1}(\inf I) \cap \partial K \subseteq K$.

Finally, the case $\varphi(y)=\inf I$ reduces to the previous one as $D$ is open and every open neighborhood of $y$ containing some $y^{\prime}$ with the property $\varphi\left(y^{\prime}\right)<\inf I$. The cases $\inf I=-\infty, \sup I \in \mathbb{R}$ and $\inf I, \sup I \in \mathbb{R}$ can be treated similarly.

Corollary 4.1. Let $C$ be a convex open subset of $\mathbb{R}^{n}$ and $K$ be a nonempty compact subset of $C$ and $\varphi: \mathbb{R}^{n} \longrightarrow \mathbb{R}$ be a linear functional. If I is some maximal open subinterval of the open set $\mathbb{R} \backslash \varphi(K)$ such that $\inf I \in \mathbb{R}$, then $\varphi^{-1}(\inf I)$ is a supporting plane of $K$. A similar statement holds for $\sup I$.

Corollary 4.2. Let $C$ be a convex open subset of $\mathbb{R}^{n}$ and $K$ be a compact convex subset of $C$. $A$ convex open subset $M$ of $C \backslash K$ is maximal in $C \backslash K$, among the convex open subsets of $C \backslash K$, if and only if $M=\varphi^{-1}(I) \cap(C \backslash K)$ for some linear functional $\varphi: \mathbb{R}^{n} \longrightarrow \mathbb{R}$ and some maximal open subinterval I of the open set $\mathbb{R} \backslash \varphi(K)$.

Proof. If $\varphi: \mathbb{R}^{n} \longrightarrow \mathbb{R}$ is a linear functional, then $\varphi^{-1}(I) \cap(C \backslash K)$ is, according to Theorem 4.1, maximal, among the convex open subsets of $C \backslash K$.

Conversely, consider a convex open subset $M$ of $C \backslash K$, which is maximal in $C \backslash K$, among the convex open subsets of $C \backslash K$. According to the Hahn-Banach separation theorem, there exists a linear functional $\varphi: \mathbb{R}^{n} \longrightarrow \mathbb{R}$ along with a real number $t$ such that $\varphi(m)<t \leq \varphi(k)$, for all $m \in M$ and all $k \in K$. We shall show that $M=\varphi^{-1}(-\infty, t) \cap(C \backslash K)$. While the inclusion $M \subseteq \varphi^{-1}(-\infty, t) \cap(C \backslash K)$ is rather obvious, it cannot be strict since $\varphi^{-1}(-\infty, t) \cap(C \backslash K)=$ $\varphi^{-1}(-\infty, t) \cap C$ is a convex open subset of $C \backslash K$, which contains $M$, and $M$ is maximal among the convex open subsets of $C \backslash K$.

Remark 4.1. Since $K$ is compact and convex in Corollary 4.2, it follows that $\varphi(K)$ is compact interval in $\mathbb{R}$, i.e., $\varphi(K)=[a, b]$, where $a=\inf \varphi(K)$ and $b=\sup \varphi(K)$. Thus, $\mathbb{R} \backslash \varphi(K)=$ $(-\infty, a) \cup(b,+\infty)$, namely, $I$ is either $(-\infty, a)$ or $(b,+\infty)$ as well as $\inf I=-\infty$ and $\sup I=a=$ $\inf \varphi(K)$ or $\inf I=b=\sup \varphi(K)$ and $\sup I=+\infty$, respectively. Replacing, if need, $\varphi$ with $-\varphi$, we may assume that $\inf I \in \mathbb{R}$ and $\sup I=\infty$, i.e. $I=(a,+\infty)$, where $a=\inf I$.

Corollary 4.3. Let $C$ be a convex open subset of $\mathbb{R}^{n}$ and $K$ be a nonempty compact subset of $C$. If $\varphi: \mathbb{R}^{n} \longrightarrow \mathbb{R}$ is a linear functional, then the set $\left(\varphi^{-1}(I) \cup X\right) \cap(C \backslash K)$ is maximal, among the convex subsets of $C \backslash K$, where I is some maximal open subinterval of the open set $\mathbb{R} \backslash \varphi(K)$ and $X$ is either a maximal convex subset of $\left(\varphi^{-1}(\inf I) \cap C\right) \backslash K$ or a maximal convex subset of $\left(\varphi^{-1}(\sup I) \cap C\right) \backslash K$ or the union of one maximal convex subset of $\left(\varphi^{-1}(\inf I) \cap C\right) \backslash K$ and one maximal convex subset of $\left(\varphi^{-1}(\sup I) \cap C\right) \backslash K$ depending on whether $\inf I \in \mathbb{R}$ and $\sup I=\infty$ or $\inf I=-\infty$ and $\sup I \in \mathbb{R}$ or $\inf I, \sup I \in \mathbb{R}$ respectively.

Proof. We first observe that $\left(\varphi^{-1}(I) \cup X\right) \cap(C \backslash K)$ is a convex subset of $C \backslash K$. If $\left(\varphi^{-1}(I) \cup X\right) \cap$ $(C \backslash K) \subseteq M$ for some convex subset $M$ of $C \backslash K$, we then deduce that $\varphi^{-1}(I) \cap(C \backslash K) \subseteq \operatorname{int}(M)$. 
Since $\varphi^{-1}(I) \cap(C \backslash K)=\varphi^{-1}(I) \cap C$ is, according with Theorem 4.1, maximal among the convex open subsets of $C \backslash K$ and $\operatorname{int}(M)$ is open, it follows that

$$
\varphi^{-1}(I) \cap(C \backslash K)=\varphi^{-1}(I) \cap C=\operatorname{int}(M) .
$$

Consequently

$$
M=\operatorname{int}(M) \cup\left(M \cap \varphi^{-1}(\inf I)\right)
$$

or

$$
M=\operatorname{int}(M) \cup\left(M \cap \varphi^{-1}(\sup I)\right)
$$

or

$$
M=\operatorname{int}(M) \cup\left(M \cap \varphi^{-1}(\inf I)\right) \cup\left(M \cap \varphi^{-1}(\sup I)\right)
$$

depending on whether $\inf I \in \mathbb{R}$ and $\sup I=\infty$ or $\inf I=-\infty$ and $\sup I \in \mathbb{R}$ or $\inf I, \sup I \in \mathbb{R}$ respectively. In the case $\inf I \in \mathbb{R}$ and $\sup I=\infty$, we have

$$
M=\operatorname{int}(M) \cup\left(M \cap \varphi^{-1}(\inf I)\right)=\varphi^{-1}(I) \cap(C \backslash K) \cup\left(M \cap \varphi^{-1}(\inf I)\right),
$$

which combined with the inclusion $\left(\varphi^{-1}(I) \cup X\right) \cap(C \backslash K) \subseteq M$ and the obvious fact $X \cap$ $\varphi^{-1}(I)=\emptyset$, leads us to the relation $X \subseteq M \cap \varphi^{-1}(\inf I)$. Thus $X$ is maximal convex subset of $\left(\varphi^{-1}(\inf I) \cap C\right) \backslash K$ and $X \subseteq M \cap \varphi^{-1}(\inf I) \subseteq\left(\varphi^{-1}(\inf I) \cap C\right) \backslash K$, which shows, via the convexity of $M \cap \varphi^{-1}(\inf I)$, that $X=M \cap \varphi^{-1}(\inf I)$ and therefore the equality $M=\left(\varphi^{-1}(I) \cup X\right) \cap$ $(C \backslash K)$. The other two cases can be treated similarly.

Corollary 4.4. Let $C$ be a convex open subset of $\mathbb{R}^{n}$ and $K$ be a compact convex subset of $C$. A convex subset $M$ of $C \backslash K$ is maximal in $C \backslash K$, among the convex subsets of $C \backslash K$, if and only if $M=\left(\varphi^{-1}(I) \cup X\right) \cap(C \backslash K)$ for some linear functional $\varphi: \mathbb{R}^{n} \longrightarrow \mathbb{R}$ and some maximal open subinterval I of the open set $\mathbb{R} \backslash \varphi(K)$, where $X$ is either a maximal convex subset of $\left(\varphi^{-1}(\inf I) \cap C\right) \backslash K$ or a maximal convex subset of $\left(\varphi^{-1}(\sup I) \cap C\right) \backslash K$, depending on whether $\inf I \in \mathbb{R}$ and $\sup I=\infty$ or $\inf I=-\infty$ and $\sup I \in \mathbb{R}$ respectively.

Proof. If $\varphi: \mathbb{R}^{n} \longrightarrow \mathbb{R}$ is a linear functional, then $\left(\varphi^{-1}(I) \cup X\right) \cap(C \backslash K)$ is, according to Corollary 4.3, maximal, among the convex subsets of $C \backslash K$.

Conversely, consider a convex subset $M$ of $C \backslash K$ which is maximal in $C \backslash K$, among the convex subsets of $C \backslash K$. According to the Hahn-Banach separation theorem, there exists a linear functional $\varphi: \mathbb{R}^{n} \longrightarrow \mathbb{R}$ along with a real number $t$ such that $\varphi(m)<t \leq \varphi(k)$, for all $m \in \operatorname{int}(M)$ and all $k \in K$. The relations $\operatorname{int}(M) \subseteq \varphi^{-1}(-\infty, t) \cap(C \backslash K)=\varphi^{-1}(-\infty, t) \cap C$ are obvious and they show that

$$
\begin{aligned}
M & =\operatorname{int}(M) \cup\left(\varphi^{-1}(t) \cap M\right) \\
& \subseteq\left(\varphi^{-1}(-\infty, t) \cap(C \backslash K)\right) \cup\left(\varphi^{-1}(t) \cap M\right) \\
& =\left(\varphi^{-1}(-\infty, t) \cup\left(\varphi^{-1}(t) \cap M\right)\right) \cap(C \backslash K) \\
& =\left(\varphi^{-1}(-\infty, t) \cup X\right) \cap(C \backslash K),
\end{aligned}
$$

where $X$ is a maximal convex subset of $\left(\varphi^{-1}(\inf I) \cap C\right) \backslash K$ which contains the convex set $\varphi^{-1}(t) \cap M$. Since $M$ is maximal among the convex subsets of $C \backslash K$ and

$$
\left(\varphi^{-1}(-\infty, t) \cup X\right) \cap(C \backslash K)=\left(\varphi^{-1}(-\infty, t) \cup X\right) \cap C
$$

is a convex subset of $C \backslash K$, it follows that $M=\left(\varphi^{-1}(-\infty, t) \cup X\right) \cap(C \backslash K)$. 
Remark 4.2. If $K$ is a nonempty compact convex subset of $\mathbb{R}^{n}$ and $\varphi: \mathbb{R}^{n} \longrightarrow \mathbb{R}$ is a linear functional, then the image $\varphi(K)$ is a compact interval, say $[a, b]$, and $\varphi^{-1}(a) \cap K, \varphi^{-1}(b) \cap K$ are faces of $K$. Indeed, we first observe that $a=\min \left(\left.\varphi\right|_{K}\right)$ and $b=\max \left(\left.\varphi\right|_{K}\right)$. Thus $\varphi^{-1}(a) \cap K$ and $\varphi^{-1}(b) \cap K$ are nonempty. Let us now consider $k_{1}, k_{2} \in K$ such that $k:=(1-t) k_{1}+t k_{2} \in$ $\varphi^{-1}(a) \cap K$, for some $t \in(0,1)$. Assume that $k_{1} \notin \varphi^{-1}(a) \cap K$, i.e. $\varphi\left(k_{1}\right)>a$. Thus

$$
\varphi\left(k_{2}\right)=\varphi\left(t^{-1}\left(k-(1-t) k_{1}\right)=t^{-1}\left(\varphi(k)-(1-t) \varphi\left(k_{1}\right)\right)<t^{-1}(a-(1-t) a)=a,\right.
$$

which is impossible since $\varphi(K)=[a, b]$. Therefore $k_{1}, k_{2} \in \varphi^{-1}(a) \cap K$.

\section{Acknowledgments}

The first author was supported by the grant of the Romanian Ministry of Research and Innovation, CNCS - UEFISCDI, project number PN-III-P4-ID-PCE-2016-0190, within PNCDI III.

\section{REFERENCES}

[1] J.W. Alexander, Functions which map the interior of the unit circle upon simple regions, Ann. Math. 17 (1915), 12-22.

[2] M. Breen, Decomposition theorems for 3-convex subsets of the plane, Pacific J. Math. 53 (1974), 43-57.

[3] M. Carmo, Differential geometry of curves and surfaces, Prentice Hall, Inc., 1976.

[4] F. Herzog, G. Piranian, On the univalence of functions whose derivative has a positive real part, Proc. Amer. Math. Soc. 2 (1951), 625-633.

[5] R.A. Horn, C.R. Johnson, Matrix Analysis, Cambridge University Press, Cambridge, 1985.

[6] D. Gale, H. Nikaidô, The Jacobian matrix and the global univalence of mappings, Math. Ann. 159 (1965), 81-93.

[7] S. Geschke, M. Kojman, W. Kubiś, R. Schipperus, Convex decompositions in the plane and continuous pair colorings of the irrationals, Israel J. Math. 131 (2002) 285-317.

[8] G. Goldman, Curvature formulas for implicit curves and surfaces, Comput. Aided Geom. Design 22 (2005), 632-658.

[9] J. Lawrence, W. Morris, Finite sets as complements of finite unions of convex sets, Discrete Comput. Geom. 42 (2009), 206-218.

[10] J.F. Lawrence, W.R. Hare, J.W. Kenell, Finite unions of convex sets, Proc. Amer. Math. Soc. 34 (1972), 225-228.

[11] R.L. McKinney, On unions of two convex sets, Canad. J. Math. 18 (1966), 883-886.

[12] D. Marian, I.R. Peter, C. Pintea, A class of generalized monotone operators, J. Math. Anal. Appl. 421 (2015), 1827-1843.

[13] J.E. Martínez-Legaz, I. Singer, Compatible Preorders and Linear Operators on $\mathbb{R}^{n}$, Linear Algebra Appl. 153 (1991), 53-66.

[14] J. Matoušek, P. Valtr, On visibility and covering by convex sets, Isreal J. Math. 113 (1999), 341-379.

[15] M. Perles, S. Shelah, A closed $(n+1)$-convex set in $\mathbb{R}^{2}$ is a union of $n$ convex sets, Israel J. Math. 70 (1990), 305-312.

[16] G. Neumann, Valence of complex-valued planar harmonic functions, Trans. Amer. Math. Soc. 357 (2005), 3133-3167.

[17] K. Noshiro, On the theory of schlicht functions, J. Fac. Sci. Hokkaido Imp. Univ. Jap. 2 (1934), 129-155.

[18] C. Pintea, Convex decompositions of convex open sets with polytopes or finite sets removed, J. Convex Anal. 26 (2019).

[19] C. Pintea, T. Trif, The monotonicity of perturbed gradients of convex functions, J. Convex Anal. 24 (2017), 525-545.

[20] S.R. Tims, A theorem on function schlicht in convex domains, Proc. London Math. Soc. S3 (1951), 200-205.

[21] F.A. Valentine, A three point convexity property, Pacific J. Math. 7 (1957), 1227-1235.

[22] S.E. Warschawski, On the higher derivatives at the boundary in conformal mapping, Trans. Amer. Math. Soc. 38 (1935), 310-340. 